\title{
Global Asymptotic Stability of a Rational System
}

\author{
Lin-Xia $\mathrm{Hu}^{1}$ and Xiu-Mei Jia ${ }^{2}$ \\ ${ }^{1}$ Department of Mathematics, Tianshui Normal University, Tianshui, Gansu 741001, China \\ ${ }^{2}$ Department of Mathematics, Hexi University, Zhangye, Gansu 734000, China
}

Correspondence should be addressed to Lin-Xia Hu; lxhu@mail.tsnc.edu.cn

Received 3 April 2014; Revised 7 July 2014; Accepted 9 July 2014; Published 7 August 2014

Academic Editor: Ziemowit Popowicz

Copyright (C) 2014 L.-X. Hu and X.-M. Jia. This is an open access article distributed under the Creative Commons Attribution License, which permits unrestricted use, distribution, and reproduction in any medium, provided the original work is properly cited.

The main goal of this paper is to investigate the global asymptotic behavior of the difference equation $x_{n+1}=\beta_{1} x_{n} /\left(A_{1}+y_{n}\right)$, $y_{n+1}=\left(\beta_{2} x_{n}+\gamma_{2} y_{n}\right) /\left(x_{n}+y_{n}\right), n=0,1,2, \ldots$ with $\beta_{1}, \beta_{2}, \gamma_{2}, A_{1} \in(0, \infty)$ and the initial value $\left(x_{0}, y_{0}\right) \in[0, \infty) \times[0, \infty)$ such that $x_{0}+y_{0} \neq 0$. The major conclusion shows that, in the case where $\gamma_{2}<\beta_{2}$, if the unique positive equilibrium $(\bar{x}, \bar{y})$ exists, then it is globally asymptotically stable.

\section{Introduction and Preliminaries}

Our aim in this paper is to investigate the dynamics of the following difference equation:

$$
\begin{array}{r}
x_{n+1}=\frac{\beta_{1} x_{n}}{A_{1}+y_{n}}, \quad y_{n+1}=\frac{\beta_{2} x_{n}+\gamma_{2} y_{n}}{x_{n}+y_{n}}, \\
n=0,1,2, \ldots
\end{array}
$$

with $\beta_{1}, \beta_{2}, \gamma_{2}, A_{1} \in(0, \infty)$ and the initial value $\left(x_{0}, y_{0}\right) \in$ $[0, \infty) \times[0, \infty)$ such that $x_{0}+y_{0} \neq 0$.

System (1) is a special case of the rational system

$$
\begin{array}{r}
x_{n+1}=\frac{\alpha_{1}+\beta_{1} x_{n}+\gamma_{1} y_{n}}{A_{1}+B_{1} x_{n}+C_{1} y_{n}}, \quad y_{n+1}=\frac{\alpha_{2}+\beta_{2} x_{n}+\gamma_{2} y_{n}}{A_{2}+B_{2} x_{n}+C_{2} y_{n}}, \\
n=0,1,2, \ldots,
\end{array}
$$

where all parameters and the initial value $\left(x_{0}, y_{0}\right)$ are nonnegative such that denominators are always positive. There is some interest in systems of rational and related difference equations, for example, see [1-9]. In this paper, we will determine the global convergence properties of the system (1) under certain conditions.
When $\beta_{2}=\gamma_{2}$, the first component $\left\{x_{n}\right\}$ of the solution $\left(x_{n}, y_{n}\right)$ of the system (1) satisfies the first-order linear difference equation

$$
x_{n+1}=\frac{\beta_{1}}{A_{1}+\beta_{2}} x_{n}, \quad n=0,1, \ldots
$$

and the second component $\left\{y_{n}\right\}$ is constant and equal to $\gamma_{2}$ for $n \geq 1$.

If the initial value is given by $x_{0}>0$, then by simple iteration, it is easy to find that

$$
x_{n}=\left(\frac{\beta_{1}}{A_{1}+\beta_{2}}\right)^{n} x_{0}
$$

is the solution of (3). If $\beta_{1}>A_{1}+\beta_{2}$, then $\lim _{n \rightarrow \infty} x_{n}=\infty$. If $\beta_{1}=A_{1}+\beta_{2}$, then $x_{n}=x_{0}$ for all $n>0$, and for $\beta_{1}<A_{1}+\beta_{2}$, we have $\lim _{n \rightarrow \infty} x_{n}=0$.

Therefore, in the remaining part, we will assume that $\beta_{2} \neq$ $\gamma_{2}$.

Clearly, $\left(0, \gamma_{2}\right)$ is always an equilibrium, and when

$$
A_{1}+\min \left\{\beta_{2}, \gamma_{2}\right\}<\beta_{1}<A_{1}+\max \left\{\beta_{2}, \gamma_{2}\right\}
$$

(1) also has a unique positive equilibrium

$$
(\bar{x}, \bar{y})=\left(\frac{\left(\beta_{1}-A_{1}\right)\left(\beta_{1}-A_{1}-\gamma_{2}\right)}{A_{1}+\beta_{2}-\beta_{1}}, \beta_{1}-A_{1}\right) .
$$


Equation (1) was investigated in [10] and the main result they obtained is the following.

Theorem 1. (i) Assume that $\gamma_{2}>\beta_{2}$. Then every solution of the system (1) converges to $\left(0, \gamma_{2}\right)$ if and only if $\beta_{1} \leq A_{1}+\beta_{2}$, and when $\beta_{1}>A_{1}+\beta_{2}$, the system (1) has unbounded solutions.

(ii) Assume that $\beta_{2}>\gamma_{2}$. Then every positive solution of the system (1) is bounded if and only if $\beta_{1}<A_{1}+\beta_{2}$. In particular, when $\beta_{1} \leq A_{1}+\gamma_{2}$, the equilibrium $\left(0, \gamma_{2}\right)$ is a global attractor of all solutions of the system (1).

In [10], the author proposed the following conjecture.

Conjecture 2. Assume that

$$
\gamma_{2}<\beta_{1}-A_{1}<\beta_{2}
$$

Show that the unique positive equilibrium $(\bar{x}, \bar{y})$ of the system (1) is globally asymptotically stable.

Inspired by Conjecture 2, we investigate the global behavior of the system (1). To start our discussion, some basic results should be presented which will be useful in the sequel.

Consider the system

$$
\begin{array}{r}
x_{n+1}=f\left(x_{n}, y_{n}\right), \quad y_{n+1}=g\left(x_{n}, y_{n}\right), \\
n=0,1,2, \ldots,
\end{array}
$$

where $F=(f, g): \mathscr{D} \rightarrow \mathbb{R}^{2}$ is continuous and $\mathscr{D} \subset \mathbb{R}^{2}$.

A vital tool for dealing with the linearized stability of (8) is the following well-known result which we incorporate in the following lemma (see, e.g., $[11,12])$.

Lemma 3. Let $F=(f, g)$ be a continuously differentiable function defined on an open set $D \subset \mathbb{R}^{2}$.

(a) If the eigenvalues of the Jacobian matrix $J_{F}((\bar{x}, \bar{y}))$ have modulus less than one, then the equilibrium of (8) is locally asymptotically stable.

(b) If at least one of the eigenvalues of the Jacobian matrix $J_{F}((\bar{x}, \bar{y}))$ has modulus greater than one, then the equilibrium of $(8)$ is unstable.

(c) The equilibrium $(\bar{x}, \bar{y})$ of (8) is locally asymptotically stable if every solution of the characteristic equation of the Jacobian matrix

$$
\lambda^{2}-p \lambda+q=0
$$

lies inside the unit circle, that is, if

$$
|p|<1+q<2 \text {. }
$$

In this case, $(\bar{x}, \bar{y})$ is also called a sink.

(d) The equilibrium $(\bar{x}, \bar{y})$ of (8) is a repeller if every solution of characteristic equation (9) lies outside the unit circle, which is equivalent to the following condition:

$$
|q|>1, \quad|p|<|1+q| .
$$

(e) The equilibrium $(\bar{x}, \bar{y})$ of (8) is a saddle point if the Jacobian matrix $J_{F}((\bar{x}, \bar{y}))$ has one eigenvalue that lies inside the unit circle and if the other one lies outside the unit circle, that is, if and only if

$$
|p|>|1+q|, \quad p^{2}-4 q>0 .
$$

The following well-known comparison result will be used in estimating the value of a solution of the system (1).

Lemma 4 (a comparison result). Assume that $\alpha \in(0, \infty)$ and $\beta \in \mathbb{R}$. Let $\left\{u_{n}\right\}_{n=0}^{\infty}$ and $\left\{v_{n}\right\}_{n=0}^{\infty}$ be sequences of real numbers such that $u_{0} \leq v_{0}$ and

$$
\begin{gathered}
u_{n+1} \leq \alpha u_{n}+\beta, \\
v_{n+1}=\alpha v_{n}+\beta, \\
n=0,1,2, \ldots
\end{gathered}
$$

Then $u_{n} \leq v_{n}$ for $n \geq 0$.

Consider the following difference equation:

$$
u_{n+1}=f\left(u_{n}\right), \quad n=0,1,2, \ldots
$$

The following result of Hautus and Bolis [13] (see also [11, 12]) deals with the global attractivity of (14).

Lemma 5. Let $I \subseteq[0, \infty)$ be some interval and assume that $f \in C[I,(0, \infty)]$ satisfies the following conditions:

(i) $f(u)$ is nondecreasing in $u$;

(ii) equation (14) has a unique positive equilibrium $\bar{u} \in I$ and the function $f(u)$ satisfies the negative feedback condition:

$$
(u-\bar{u})(f(u)-u)<0 \quad \text { for every } u \in I \backslash\{\bar{u}\}
$$

Then every positive solution of (14) with initial conditions in I converges to $\bar{u}$.

To prepare for our major investigation, we consider the following equation:

$$
u_{n+1}=g\left(u_{n}, u_{n-1}\right), \quad n=0,1,2, \ldots,
$$

and the following lemma should be mentioned which is from [12].

Lemma 6. Let $[a, b]$ be an interval of real numbers and assume that $g:[a, b]^{2} \rightarrow[a, b]$ is a continuous function satisfying the following properties:

(i) $g(x, y)$ is nondecreasing in each of its arguments;

(ii) the function $g(m, m)=m$ has a unique positive solution.

Then (16) has a unique equilibrium $\bar{u} \in[a, b]$ and every solution of (16) converges to $\bar{u}$. 


\section{Linearized Stability}

In this section, we will make some conclusions about linearized stability. Consider the map $T$ on $\mathbb{R}^{2}$ associated with the system (1), that is,

$$
T(x, y)=\left(\begin{array}{c}
f_{1}(x, y) \\
f_{2}(x, y)
\end{array}\right)=\left(\begin{array}{c}
\frac{\beta_{1} x}{A_{1}+y} \\
\frac{\beta_{2} x+\gamma_{2} y}{x+y}
\end{array}\right) .
$$

Calculating the partial derivatives of the functions $f_{1}(x, y)$ and $f_{2}(x, y)$ shows that

$$
\begin{array}{cc}
\frac{\partial f_{1}}{\partial x}=\frac{\beta_{1}}{A_{1}+y}, & \frac{\partial f_{1}}{\partial y}=-\frac{\beta_{1} x}{\left(A_{1}+y\right)^{2}}, \\
\frac{\partial f_{2}}{\partial x}=\frac{\left(\beta_{2}-\gamma_{2}\right) y}{(x+y)^{2}}, & \frac{\partial f_{2}}{\partial y}=\frac{\left(\gamma_{2}-\beta_{2}\right) x}{(x+y)^{2}} .
\end{array}
$$

The Jacobian matrix of $T$ evaluated at $\left(0, \gamma_{2}\right)$ is

$$
J_{F}\left(\left(0, \gamma_{2}\right)\right)=\left(\begin{array}{cc}
\frac{\beta_{1}}{A_{1}+\gamma_{2}} & 0 \\
\frac{\beta_{2}-\gamma_{2}}{\gamma_{2}} & 0
\end{array}\right)
$$

and its eigenvalues are $\lambda_{1}=0$ and $\lambda_{2}=\beta_{1} /\left(A_{1}+\gamma_{2}\right)$.

Another equilibrium $(\bar{x}, \bar{y})$, namely, $(6)$, exists if and only if (5) holds. Using the equality $A_{1}+\bar{y}=\beta_{1}$, the Jacobian matrix of $T$ evaluated at $(\bar{x}, \bar{y})$ is

$$
J_{F}((\bar{x}, \bar{y}))=\left(\begin{array}{cc}
1 & -\frac{\bar{x}}{\beta_{1}} \\
\frac{\left(\beta_{2}-\gamma_{2}\right) \bar{y}}{(\bar{x}+\bar{y})^{2}} & \frac{\left(\gamma_{2}-\beta_{2}\right) \bar{x}}{(\bar{x}+\bar{y})^{2}}
\end{array}\right),
$$

and its characteristic equation associated with $(\bar{x}, \bar{y})$ is given by

$$
\lambda^{2}-p \lambda+q=0
$$

where

$$
\begin{aligned}
p & =1+\frac{\left(\gamma_{2}-\beta_{2}\right) \bar{x}}{(\bar{x}+\bar{y})^{2}} \\
& =\frac{\left(\beta_{1}-A_{1}\right)\left(\beta_{2}-\gamma_{2}\right)-\left(\beta_{1}-A_{1}-\gamma_{2}\right)\left(A_{1}+\beta_{2}-\beta_{1}\right)}{\left(\beta_{1}-A_{1}\right)\left(\beta_{2}-\gamma_{2}\right)}, \\
q & =\frac{\left(\beta_{2}-\gamma_{2}\right) \bar{x}\left(\bar{y}-\beta_{1}\right)}{\beta_{1}(\bar{x}+\bar{y})^{2}} \\
& =-\frac{A_{1}\left(\beta_{1}-A_{1}-\gamma_{2}\right)\left(A_{1}+\beta_{2}-\beta_{1}\right)}{\beta_{1}\left(\beta_{1}-A_{1}\right)\left(\beta_{2}-\gamma_{2}\right)} .
\end{aligned}
$$

When $\beta_{2}>\gamma_{2}$, we find that $\gamma_{2}<\beta_{1}-A_{1}<\beta_{2}$ and $A_{1} / \beta_{1}<1$. Thus $p>0$ and

$$
\begin{aligned}
1+q & =1-\frac{A_{1}\left(\beta_{1}-A_{1}-\gamma_{2}\right)\left(A_{1}+\beta_{2}-\beta_{1}\right)}{\beta_{1}\left(\beta_{1}-A_{1}\right)\left(\beta_{2}-\gamma_{2}\right)} \\
& >1-\frac{\left(\beta_{1}-A_{1}-\gamma_{2}\right)\left(A_{1}+\beta_{2}-\beta_{1}\right)}{\left(\beta_{1}-A_{1}\right)\left(\beta_{2}-\gamma_{2}\right)}=|p|, \\
0>q & =-\frac{A_{1}\left(\beta_{1}-A_{1}-\gamma_{2}\right)\left(A_{1}+\beta_{2}-\beta_{1}\right)}{\beta_{1}\left(\beta_{1}-A_{1}\right)\left(\beta_{2}-\gamma_{2}\right)} \\
& =-\frac{A_{1}}{\beta_{1}} \frac{\beta_{1}-A_{1}-\gamma_{2}}{\beta_{1}-A_{1}} \cdot \frac{\beta_{2}-\left(\beta_{1}-A_{1}\right)}{\beta_{2}-\gamma_{2}}>-1 .
\end{aligned}
$$

When $\gamma_{2}>\beta_{2}, A_{1} / \beta_{1}<1$ holds and by simple computation, we have

$$
\begin{aligned}
p^{2}-4 q & =\left[1+\frac{\left(\gamma_{2}-\beta_{2}\right) \bar{x}}{(\bar{x}+\bar{y})^{2}}\right]^{2}-4 \frac{\left(\beta_{2}-\gamma_{2}\right) \bar{x}\left(\bar{y}-\beta_{1}\right)}{\beta_{1}(\bar{x}+\bar{y})^{2}} \\
& >\left[1+\frac{\left(\gamma_{2}-\beta_{2}\right) \bar{x}}{(\bar{x}+\bar{y})^{2}}\right]^{2}-4 \frac{\left(\gamma_{2}-\beta_{2}\right) \bar{x}}{(\bar{x}+\bar{y})^{2}} \\
& =\left[1-\frac{\left(\gamma_{2}-\beta_{2}\right) \bar{x}}{(\bar{x}+\bar{y})^{2}}\right]^{2} \geq 0 .
\end{aligned}
$$

Furthermore, $p, q>0$ and

$$
|1+q|=1+\frac{A_{1}\left(\gamma_{2}-\beta_{2}\right) \bar{x}}{\beta_{1}(\bar{x}+\bar{y})^{2}}<1+\frac{\left(\gamma_{2}-\beta_{2}\right) \bar{x}}{(\bar{x}+\bar{y})^{2}}=|p| .
$$

Employing Lemma 3, we formulate the results in the following.

Theorem 7. (i) The equilibrium $\left(0, \gamma_{2}\right)$ of the system (1) is locally asymptotically stable when $\beta_{1}-A_{1}<\gamma_{2}$, and it is unstable (a saddle point) when $\beta_{1}-A_{1}>\gamma_{2}$, and it is nonhyperbolic when $\beta_{1}-A_{1}=\gamma_{2}$.

(ii) Assume that $\gamma_{2}<\beta_{2}$ and (7) holds. Then the unique positive equilibrium $(\bar{x}, \bar{y})$ of the system (1) is locally asymptotically stable.

(iii) Assume that $\beta_{2}<\gamma_{2}$ and $\beta_{2}<\beta_{1}-A_{1}<\gamma_{2}$. Then the unique positive equilibrium $(\bar{x}, \bar{y})$ of the system (1) is unstable; further, it is a saddle point.

\section{Global Attractivity}

In this section, we will commence global asymptotic stability analysis. Let $\left(x_{n}, y_{n}\right)$ be a solution of the system (1), then it is easy to obtain the following result from the second equation of the system (1).

Theorem 8. (i) Assume that $\beta_{2}>\gamma_{2}$. Then every solution $\left(x_{n}, y_{n}\right)$ of the system (1) satisfies $\gamma_{2} \leq y_{n} \leq \beta_{2}$ for $n \geq 1$.

(ii) Assume that $\gamma_{2}>\beta_{2}$. Then every solution $\left(x_{n}, y_{n}\right)$ of the system (1) satisfies $\beta_{2} \leq y_{n} \leq \gamma_{2}$ for $n \geq 1$. 
Theorem 9. Every solution of the system (1) with $x_{0}=0$ converges to $\left(0, \gamma_{2}\right)$.

Proof. Since $x_{0}=0$ implies that $x_{n}=0$ for $n \geq 1$, thus $\lim _{n \rightarrow \infty} y_{n}=\gamma_{2}$, finishing the proof.

Theorem 10. Assume that $\gamma_{2}<\beta_{2} \leq \beta_{1}-A_{1}$. Then every solution of the system (1) with $x_{0}>0$ satisfies $\lim _{n \rightarrow \infty} x_{n}=\infty$, $\lim _{n \rightarrow \infty} y_{n}=\beta_{2}$.

Proof. Using Theorem 8, we get that when $\gamma_{2}<\beta_{2}<\beta_{1}-A_{1}$,

$$
x_{n+1}=\frac{\beta_{1}}{A_{1}+y_{n}} x_{n}>\frac{\beta_{1}}{A_{1}+\beta_{2}} x_{n} \rightarrow \infty,
$$

and when $\gamma_{2}<\beta_{2}=\beta_{1}-A_{1}$,

$$
x_{n+1}=\frac{\beta_{1}}{A_{1}+y_{n}} x_{n}>\frac{\beta_{1}}{A_{1}+\beta_{2}} x_{n}=x_{n} \rightarrow \infty,
$$

since the only equilibrium of the system $(1)$ is $\left(0, \gamma_{2}\right)$ when $\beta_{1}=A_{1}+\beta_{2}$.

Further, using the boundedness of $y_{n}$, we have

$$
y_{n+1}=\frac{\beta_{2} x_{n}+\gamma_{2} y_{n}}{x_{n}+y_{n}}=\frac{\beta_{2}+\gamma_{2}\left(y_{n} / x_{n}\right)}{1+\left(y_{n} / x_{n}\right)} \rightarrow \beta_{2} .
$$

The proof is complete.

For the case where $\beta_{1} \leq A_{1}+\gamma_{2}$, the authors had obtained that the unique positive equilibrium $\left(0, \gamma_{2}\right)$ is a global attractor of all solutions of the system (1) in [10], see Theorem 1 (ii). Moreover, in view of Theorem 7 (i), we may formulate the result in the following theorem.

Theorem 11. Assume that $\beta_{1}-A_{1}<\gamma_{2}<\beta_{2}$. Then the unique equilibrium $\left(0, \gamma_{2}\right)$ of the system (1) is globally asymptotically stable.

Now, we pay attention to dealing with the global attractivity of the unique positive equilibrium $(\bar{x}, \bar{y})$, namely, (6), under the condition that $\gamma_{2}<\beta_{2}$. In this case, $(\bar{x}, \bar{y})$ exists if and only if (7) holds. To obtain the global attractivity of $(\bar{x}, \bar{y})$, the following useful lemma should first be established.

Consider the following difference equation:

$$
u_{n+1}=a \frac{u_{n}\left(u_{n}+1\right)}{u_{n}+b}, \quad n=1,2, \ldots
$$

where $0<b<a<1$ and the initial value $u_{1}=x_{1} / y_{1}=$ $\beta_{1} x_{0}\left(x_{0}+y_{0}\right) /\left(A_{1}+y_{0}\right)\left(\beta_{2} x_{0}+\gamma_{2} y_{0}\right)$. Equation (30) possesses two equilibria, namely, zero and $\bar{u}=(a-b) /(1-a)$.

Lemma 12. Every positive solution of (30) converges to the unique positive equilibrium $\bar{u}$.
Proof. Clearly, $u_{0}>0$ implies that $u_{n}>0$ for $n \geq 1$. Let $f(u)=a u(u+1) /(u+b)$, then $f(u)$ is increasing in $u$ for $u>0$ and

$$
\begin{aligned}
(u-\bar{u})\left[\frac{a u(u+1)}{u+b}-u\right] & =u(u-\bar{u})\left[\frac{a-b-(1-a) u}{u+b}\right] \\
& =-(1-a) \frac{u(u-\bar{u})^{2}}{u+b}<0 .
\end{aligned}
$$

Thus $\lim _{n \rightarrow \infty} u_{n}=\bar{u}$ for $u_{0}>0$ by applying Lemma 5 .

The proof is complete.

Theorem 13. Assume that (7) holds. Then the unique positive equilibrium $(\bar{x}, \bar{y})$ of the system (1) is globally asymptotically stable.

Proof. In view of Theorem 7 , it is sufficient to show that $(\bar{x}, \bar{y})$ is a global attractor of all positive solutions of the system (1).

In this case, $y_{n} \geq \gamma_{2}>0$ holds for $n \geq 1$ and thus the system (1) yields

$$
\begin{gathered}
\frac{x_{n+1}}{y_{n+1}}=\frac{\beta_{1}\left(x_{n} / y_{n}\right)}{1+\left(A_{1} / y_{n}\right)} \frac{\left(x_{n} / y_{n}\right)+1}{\beta_{2}\left(x_{n} / y_{n}\right)+\gamma_{2}} \\
=\frac{\beta_{1}}{\beta_{2}\left(1+\left(A_{1} / y_{n}\right)\right)} \frac{\left(x_{n} / y_{n}\right)\left(\left(x_{n} / y_{n}\right)+1\right)}{\left(x_{n} / y_{n}\right)+\left(\gamma_{2} / \beta_{2}\right)}, \\
y_{n+1}=\frac{\beta_{2}\left(x_{n} / y_{n}\right)+\gamma_{2}}{\left(x_{n} / y_{n}\right)+1},
\end{gathered}
$$

for $n \geq 1$. Let $u_{n}=x_{n} / y_{n}, v_{n}=y_{n}$, then the system (1) becomes

$$
\begin{aligned}
u_{n+1}=\frac{\beta_{1}}{\beta_{2}\left(1+\left(A_{1} / v_{n}\right)\right)} \frac{u_{n}\left(u_{n}+1\right)}{u_{n}+\left(\gamma_{2} / \beta_{2}\right)}, \quad v_{n+1} & =\frac{\beta_{2} u_{n}+\gamma_{2}}{u_{n}+1}, \\
n & =1,2, \ldots
\end{aligned}
$$

Further, the system (33) may reduce to the following second-order difference equation:

$$
\begin{array}{r}
u_{n+1}=\frac{\beta_{1} u_{n}\left(u_{n}+1\right)}{\beta_{2} u_{n}+\gamma_{2}} \frac{\beta_{2} u_{n-1}+\gamma_{2}}{\beta_{2} u_{n-1}+\gamma_{2}+A_{1}\left(u_{n-1}+1\right)}, \\
n=2,3, \ldots .
\end{array}
$$

Clearly, zero is always the equilibrium of (34) and when (7) holds, (34) also possesses a unique positive equilibrium

$$
\bar{u}=\frac{\beta_{1}-A_{1}-\gamma_{2}}{A_{1}+\beta_{2}-\beta_{1}} .
$$

Notice that $\gamma_{2} \leq v_{n}=y_{n} \leq \beta_{2}$ for $n \geq 1$, and we get

$$
\frac{\beta_{1} \gamma_{2}}{\beta_{2}\left(A_{1}+\gamma_{2}\right)} \leq \frac{\beta_{1}}{\beta_{2}\left(1+\left(A_{1} / v_{n}\right)\right)} \leq \frac{\beta_{1}}{A_{1}+\beta_{2}},
$$

and thus

$$
\frac{\beta_{1} \gamma_{2}}{\beta_{2}\left(A_{1}+\gamma_{2}\right)} \frac{u_{n}\left(u_{n}+1\right)}{u_{n}+\left(\gamma_{2} / \beta_{2}\right)}
$$




$$
\begin{aligned}
& \leq u_{n+1}=\frac{\beta_{1}}{\beta_{2}\left(1+\left(A_{1} / v_{n}\right)\right)} \frac{u_{n}\left(u_{n}+1\right)}{u_{n}+\left(\gamma_{2} / \beta_{2}\right)} \\
& \leq \frac{\beta_{1}}{A_{1}+\beta_{2}} \frac{u_{n}\left(u_{n}+1\right)}{u_{n}+\left(\gamma_{2} / \beta_{2}\right)}, \quad n \geq 1 .
\end{aligned}
$$

Let $a=\beta_{1} \gamma_{2} / \beta_{2}\left(A_{1}+\gamma_{2}\right), b=\gamma_{2} / \beta_{2}$, then $a<$ $\left(\beta_{1} / \beta_{2}\right)\left(\beta_{2} /\left(A_{1}+\beta_{2}\right)\right)=\beta_{1} /\left(A_{1}+\beta_{2}\right)<1$ and $b<a<1$. Hence by Lemma 12, we get that every positive solution of the following difference equation

$$
\breve{u}_{n+1}=\frac{\beta_{1} \gamma_{2}}{\beta_{2}\left(A_{1}+\gamma_{2}\right)} \frac{\breve{u}_{n}\left(\breve{u}_{n}+1\right)}{\breve{u}_{n}+\left(\gamma_{2} / \beta_{2}\right)}, \quad n=1,2, \ldots
$$

converges to its unique positive equilibrium $\breve{u}=\gamma_{2}\left(\beta_{1}-\left(A_{1}+\right.\right.$ $\left.\left.\gamma_{2}\right)\right) /\left(\beta_{2} A_{1}+\gamma_{2}\left(\beta_{2}-\beta_{1}\right)\right)$.

Let $a=\beta_{1} /\left(A_{1}+\beta_{2}\right), b=\gamma_{2} / \beta_{2}$, then $\gamma_{2} / \beta_{2}<\left(A_{1}+\right.$ $\left.\gamma_{2}\right) /\left(A_{1}+\beta_{2}\right)<\beta_{1} /\left(A_{1}+\beta_{2}\right)<1$, which means that $b<a<1$. Similarly, by Lemma 12, we know that every positive solution of the following difference equation

$$
\widehat{u}_{n+1}=\frac{\beta_{1}}{A_{1}+\beta_{2}} \frac{\widehat{u}_{n}\left(\widehat{u}_{n}+1\right)}{\widehat{u}_{n}+\left(\gamma_{2} / \beta_{2}\right)}, \quad n=1,2, \ldots
$$

converges to its unique positive equilibrium $\widehat{u}=\left(\beta_{1} \beta_{2}-\right.$ $\left.\gamma_{2}\left(A_{1}+\beta_{2}\right)\right) / \beta_{2}\left(A_{1}+\beta_{2}-\beta_{1}\right)$.

Applying Lemma 4 and (37), we find that every solution of (34) with initial value $\breve{u}_{1}=\widehat{u}_{1}=u_{1}=\left(x_{1} / y_{1}\right)>0$ satisfies

$$
\breve{u}_{n} \leq u_{n} \leq \widehat{u}_{n}, \quad \text { for } n \geq 1 \text {. }
$$

Hence for $0<\epsilon<\breve{u} / 2$, there exists an integer $N$ such that for $n>N$,

$$
\frac{\breve{u}}{2}<\breve{u}-\epsilon<\breve{u}_{n} \leq u_{n} \leq \widehat{u}_{n}<\widehat{u}+\epsilon<\widehat{u}+\frac{\breve{u}}{2} .
$$

Moreover,

$$
\frac{\breve{u}}{2} \leq \liminf _{n \rightarrow \infty} u_{n} \leq \limsup _{n \rightarrow \infty} u_{n} \leq \widehat{u}+\frac{\breve{u}}{2} .
$$

Let $I=\breve{u} / 2, S=\widehat{u}+(\breve{u} / 2)$, then $S>I>0$ and every solution of (34) eventually enters the invariant interval $[I, S]$.

Denote the function

$$
g(u, v)=\frac{\beta_{1} u(u+1)}{\beta_{2} u+\gamma_{2}} \frac{\beta_{2} v+\gamma_{2}}{\beta_{2} v+\gamma_{2}+A_{1}(v+1)},
$$

and simple computation shows that

$$
\begin{gathered}
\frac{\partial g}{\partial u}=\frac{\beta_{1}\left(\beta_{2} u^{2}+2 \gamma_{2} u+\gamma_{2}\right)}{\left(\beta_{2} u+\gamma_{2}\right)^{2}} \frac{\beta_{2} v+\gamma_{2}}{\beta_{2} v+\gamma_{2}+A_{1}(v+1)}>0 \\
\frac{\partial g}{\partial v}=\frac{\beta_{1} u(u+1)}{\beta_{2} u+\gamma_{2}} \frac{A_{1}\left(\beta_{2}-\gamma_{2}\right)}{\left[\beta_{2} v+\gamma_{2}+A_{1}(v+1)\right]^{2}}>0
\end{gathered}
$$

Applying Lemma 6, to establish the global attractivity of the equilibrium $\bar{u}$ of (34), it is sufficient to confirm that the following equation

$$
g(m, m)=\frac{\beta_{1} m(m+1)}{\beta_{2} m+\gamma_{2}} \frac{\beta_{2} m+\gamma_{2}}{\beta_{2} m+\gamma_{2}+A_{1}(m+1)}=m
$$

has a unique positive solution.

Solving (46), we get

$$
\beta_{1}(m+1)=\beta_{2} m+\gamma_{2}+A_{1}(m+1),
$$

from which it follows that

$$
m=\frac{\beta_{1}-A_{1}-\gamma_{2}}{A_{1}+\beta_{2}-\beta_{1}}=\bar{u} .
$$

Therefore, $\lim _{n \rightarrow \infty} u_{n}=\bar{u}$, and hence,

$$
\lim _{n \rightarrow \infty} y_{n}=\lim _{n \rightarrow \infty} v_{n}=\frac{\beta_{2} \bar{u}+\gamma_{2}}{\bar{u}+1}=\beta_{1}-A_{1}=\bar{y} .
$$

Furthermore,

$$
\begin{aligned}
\lim _{n \rightarrow \infty} x_{n} & =\lim _{n \rightarrow \infty} u_{n} \cdot \lim _{n \rightarrow \infty} y_{n}=\bar{u} \cdot \bar{y} \\
& =\frac{\left(\beta_{1}-A_{1}\right)\left(\beta_{1}-A_{1}-\gamma_{2}\right)}{A_{1}+\beta_{2}-\beta_{1}}=\bar{x},
\end{aligned}
$$

and thus the result follows.

The proof is complete.

\section{Conflict of Interests}

The authors declare that there is no conflict of interests regarding the publication of this paper.

\section{References}

[1] E. Camouzis, M. R. S. Kulenović, G. Ladas, and O. Merino, "Rational systems in the plane," Journal of Difference Equations and Applications, vol. 15, no. 3, pp. 303-323, 2009.

[2] B. D. Iričanin and S. Stević, "Some systems of nonlinear difference equations of higher order with periodic solutions," Dynamics of Continuous, Discrete and Impulsive Systems, vol. 13, no. 3-4a, pp. 499-508, 2006.

[3] G. Lugo and F. J. Palladino, "On the boundedness character of rational systems in the plane," Journal of Difference Equations and Applications, vol. 17, no. 12, pp. 1801-1811, 2011.

[4] G. Papaschinopoulos and C. J. Schinas, "On a system of two nonlinear difference equations," Journal of Mathematical Analysis and Applications, vol. 219, no. 2, pp. 415-426, 1998.

[5] S. Stević, "On a system of difference equations with period two coefficients," Applied Mathematics and Computation, vol. 218, no. 8, pp. 4317-4324, 2011.

[6] S. Stević, "On some solvable systems of difference equations," Applied Mathematics and Computation, vol. 218, no. 9, pp. 50105018, 2012.

[7] S. Stević, "On the difference equation $x_{n}=x_{n-2} /\left(b_{n}+\right.$ $\left.c_{n} x_{n-1} x_{n-2}\right)$," Applied Mathematics and Computation, vol. 218, no. 8, pp. 4507-4513, 2011. 
[8] S. Stević, J. Diblík, B. Iričanin, and Z. Šmarda, "On a periodic system of difference equations," Abstract and Applied Analysis, vol. 2012, Article ID 258718, 5 pages, 2012.

[9] S. Stević, J. Diblík, B. Iričanin, and Z. Šmarda, "On some solvable difference equations and systems of difference equations," Abstract and Applied Analysis, vol. 2012, Article ID 541761, 11 pages, 2012.

[10] E. Camouzis and G. Ladas, "Global results on rational systems in the plane, part 1," Journal of Difference Equations and Applications, vol. 16, no. 8, pp. 975-1013, 2010.

[11] V. L. Kocic and G. Ladas, Global Behavior of Nonlinear Difference Equations of Higher Order with Application, Kluwer Academic, Dordrecht, The Netherlands, 1993.

[12] M. R. S. Kulenović and G. Ladas, Dynamics of Second Order Rational Difference Equations with Open Problems and Conjectures, Chapman Hall/CRC, Boca Raton, Fla, USA, 2002.

[13] M. L. J. Hautus and T. S. Bolis, "Solution to problem E2721," American Mathematical Monthly, vol. 86, pp. 865-866, 1979. 


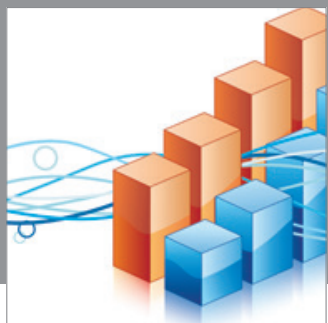

Advances in

Operations Research

mansans

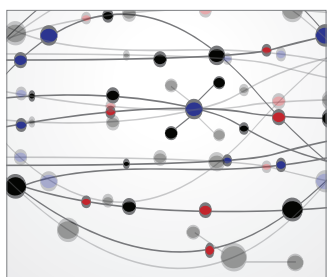

The Scientific World Journal
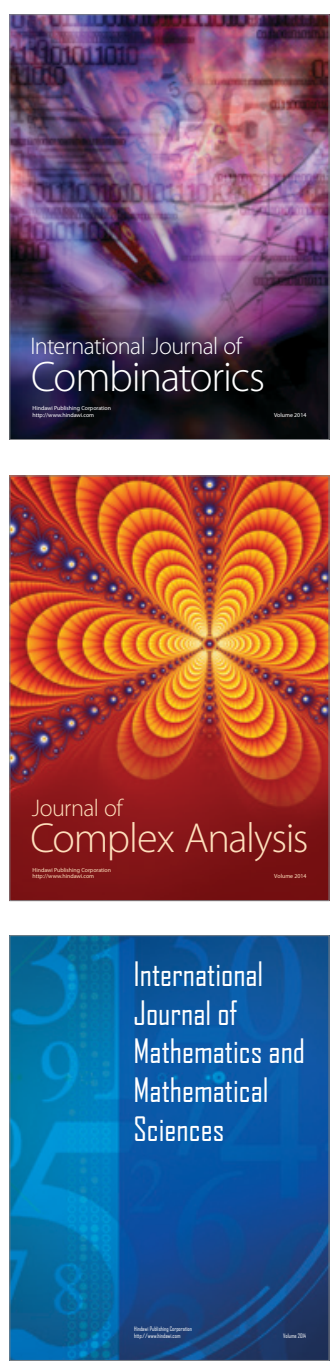
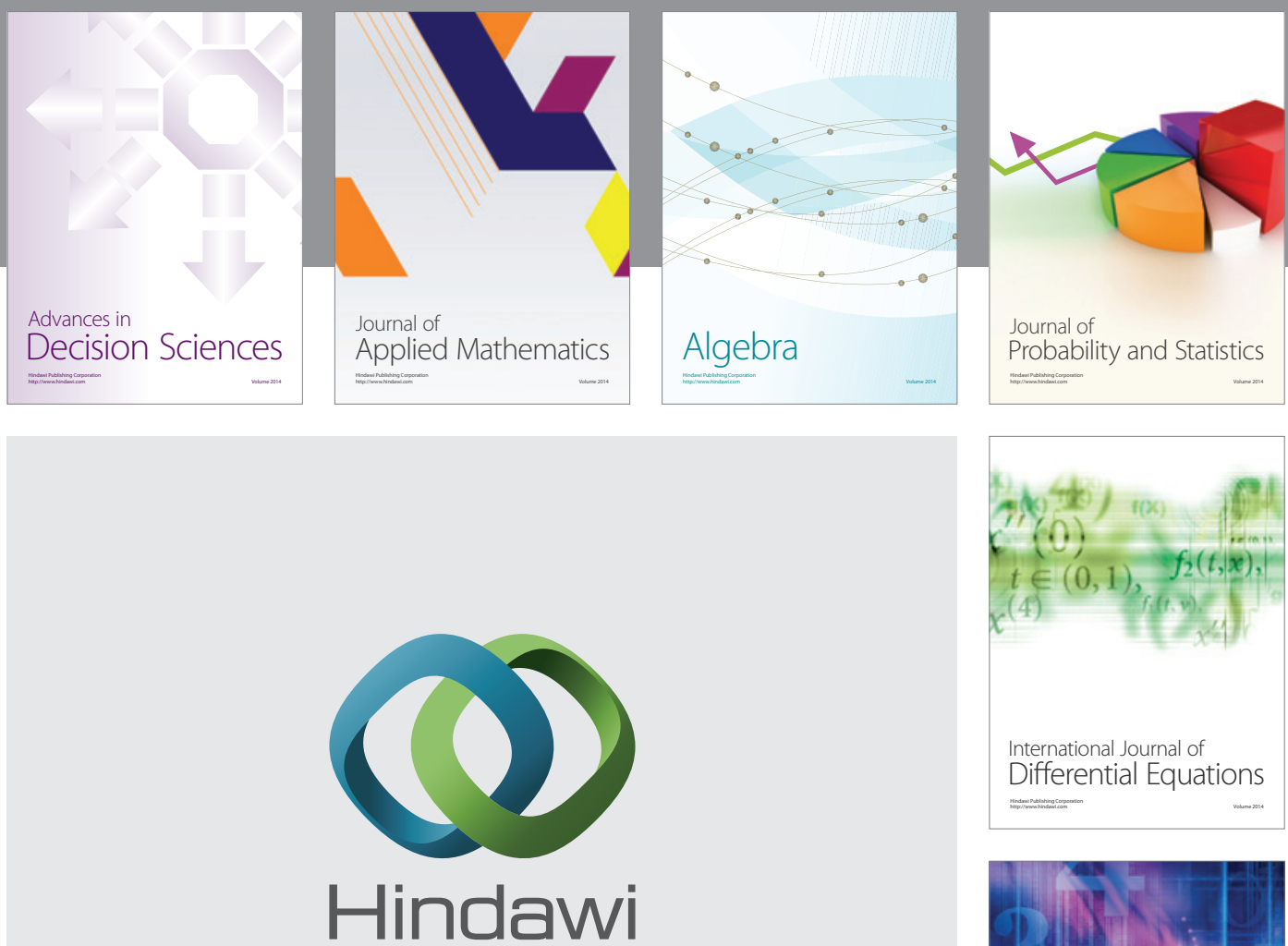

Submit your manuscripts at http://www.hindawi.com
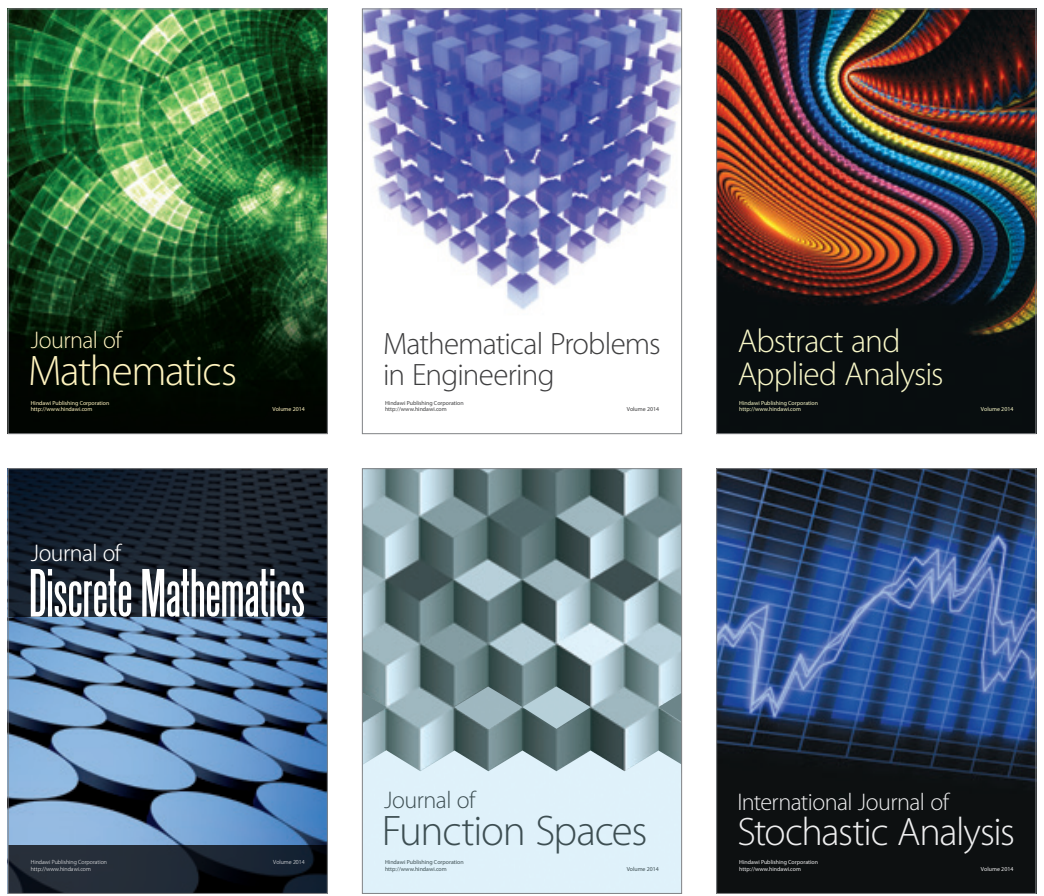

Journal of

Function Spaces

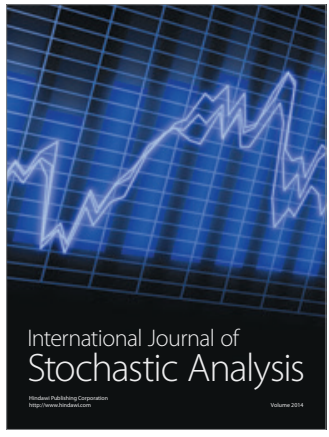

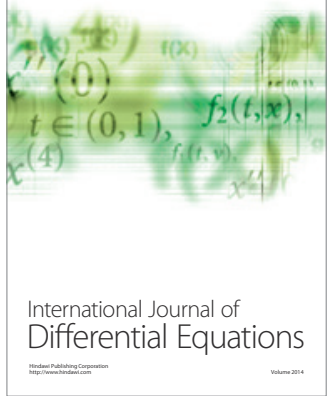
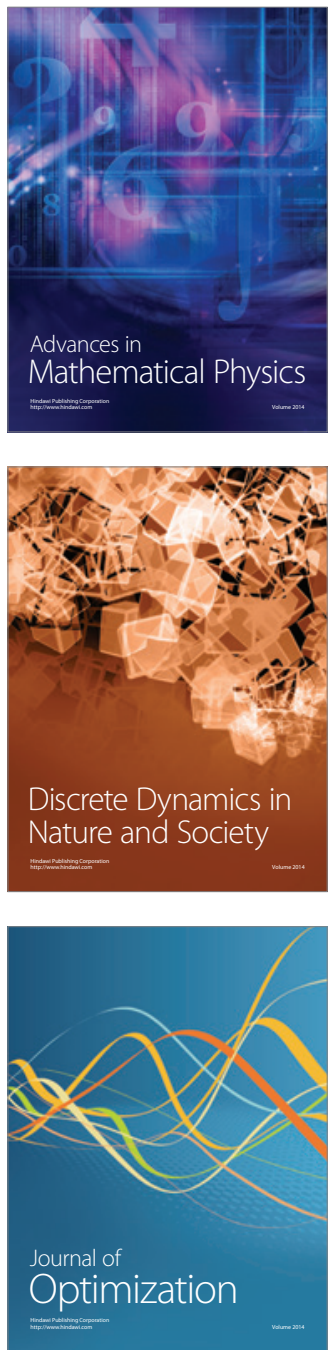